\title{
ANÁLISE DO PERFIL PROFISSIONAL DE CIRURGIÕES-DENTISTAS GRADUADOS NA FACULDADE DE ODONTOLOGIA DE BAURU - USP ENTRE OS ANOS DE 1996 E 2000
}

\author{
PROFESSIONAL PROFILE ANALYSIS OF DENTISTS GRADUATED AT BAURU \\ DENTAL SCHOOL - UNIVERSITY OF SÃO PAULO BETWEEN 1996 AND 2000
}

\author{
José Roberto de Magalhães BASTOS \\ Professor Titular do Departamento de Odontopediatria, Ortodontia e Saúde Coletiva da Faculdade de Odontologia de Bauru da Universidade \\ de São Paulo (FOB-USP).
}

\section{Aline Guerra AQUILANTE}

Beatriz Simões de ALMEIDA

Especialistas em Odontologia em Saúde Coletiva pela Associação Paulista de Cirurgiões-Dentistas (APCD) - Regional Bauru.

\section{José Roberto Pereira LAURIS}

Professor Doutor do Departamento de Odontopediatria, Ortodontia e Saúde Coletiva da FOB-USP.

\section{Vitoriano Truvijo BIJELLA}

Professor Titular do Departamento de Odontopediatria, Ortodontia e Saúde Coletiva da FOB-USP.

\begin{abstract}
$O$ S objetivos do presente estudo foram determinar o perfil profissional dos 248 cirurgiões-dentistas graduados na Faculdade de Odontologia de Bauru - USP entre 1996 e 2000, constatar se há a adoção de medidas educativopreventivas de caráter coletivo por parte destes profissionais e determinar o grau de satisfação profissional, através de um questionário contendo questões abertas e de múltipla escolha. O índice de retorno foi de 39,5\%. A análise dos resultados revelou que os cirurgiões-dentistas eram predominantemente do gênero masculino, escolheram a Odontologia como profissão por afinidade e consideraram bom o curso de Graduação. Os resultados demonstraram que os profissionais ainda não despertaram para a importância da educação e da prevenção, principalmente em nível coletivo. Concluiu-se que o perfil predominante do profissional é aquele que trabalha no consultório particular próprio $(38,8 \%)$ ou por porcentagem (25,5\%), sendo que $26,5 \%$ atende em algum Convênio ou Cooperativa e apenas $12,2 \%$ trabalham na rede pública. Embora $63,3 \%$ tenham afirmado que se sentem realizados profissionalmente, apenas $12,2 \%$ estão financeiramente realizados. Uma das maiores dificuldades enfrentadas pelos cirurgiões-dentistas é a alta competitividade e saturação do mercado de trabalho.
\end{abstract}

UNITERMOS: Recursos humanos em odontologia; Prática profissional; Odontólogos; Questionários.

\section{INTRODUÇÃO}

O Brasil apresenta uma proporção de 1.020 cirurgiõesdentistas por habitante, melhor inclusive do que a recomendada pela Organização Mundial de Saúde, que é de 1 cirurgião-dentista para 1.200 habitantes. O problema é que a distribuição de dentistas nos centros urbanos e no interior é bastante desequilibrada, concentrando-se nas regiões de maior renda, com destaque para as clínicas particulares. São Paulo e Rio de Janeiro apresentam, por exemplo, proporções de 1/ 635 e 1/713, respectivamente, enquanto o Maranhão oferece à população somente 1 cirurgião-dentista para 4.941 habitantes ${ }^{13}$.

A pouca procura por tratamento odontológico se deve à perda do poder aquisitivo por parte da população, que nos últimos anos provocou uma "fuga" dos consultórios. Com o salário mínimo fixado em torno de $\mathrm{R} \$ 200,00$, dificilmente a maioria da população tem condições de procurar tratamento dentário. A partir do momento em que os consultórios ficam "vazios", o trabalho autônomo perde força e dá lugar ao trabalho assalariado direto (contratação) ou indireto (credenciamento). Essa inversão promove mudanças no exercício profissional e também exige a busca de alterações de postura diante dessas novas situações ${ }^{13}$. 
$\operatorname{Costa}^{3}$ (1988) estudou o papel da formação universitária no desempenho profissional dos dentistas da Grande São Paulo e constatou que embora a Faculdade proporcione uma sólida formação teórica, a maioria dos dentistas sugere aumento da carga horária curricular destinada ao treinamento prático e criticam o ensino, argumentando que este é ilustrativo, superficial e não corresponde às necessidades terapêuticas.

Stewart, Ralph, Macmillan ${ }^{16,17,18}(1989,1990)$, realizaram um levantamento sobre as opiniões dos estudantes de Odontologia da Universidade de Melbourne e dos dentistas registrados na Dental Board e residentes no estado de Victoria, Austrália. Questionamentos foram realizados a respeito da formação universitária e da situação após o Curso de Graduação. A maioria dos profissionais era do gênero masculino, trabalhava em consultório particular e sugeriu que a carga horária do curso fosse aumentada.

Falasco, Robinson, Faja ${ }^{7}$, em 1990, já ressaltavam os problemas encontrados pelos recém-formados da Faculdade de Odontologia da Universidade de Michigan para se estabelecerem na prática da Odontologia.

Às vésperas do século XXI, Marcelino ${ }^{13}$, em 2000, afirmou que uma série de mudanças na organização da produção e do trabalho marca a fase atual da Odontologia; assim, faz-se necessária uma adequação da política de recursos humanos para a construção deste novo modelo de atenção à saúde. Devido a isto, através de um questionário contendo questões abertas e de múltipla escolha, realizou um trabalho com o objetivo de analisar o perfil de cirurgiões-dentistas do município de Araçatuba-SP e a sua percepção social frente às mudanças ocorridas na Odontologia.

Assim, os objetivos do presente trabalho foram determinar o perfil profissional dos cirurgiões-dentistas graduados na Faculdade de Odontologia de Bauru - USP entre os anos de 1996 e 2000, constatar se há a adoção de medidas educativopreventivas de caráter coletivo por parte destes profissionais e determinar o grau de satisfação profissional.

\section{MATERIAIS E MÉTODOS}

O material utilizado na pesquisa foi um questionário, baseado em estudos ${ }^{3,13}$ de metodologia semelhante. O questionário foi enviado, uma única vez, aos 248 cirurgiõesdentistas graduados na Faculdade de Odontologia de Bauru (FOB) - USP entre os anos de 1996 e 2000, contendo questões abertas e de múltipla escolha. Para facilitar a resposta e devolução dos questionários, dentro do envelope enviado aos cirurgiões-dentistas havia um envelope selado e endereçado ao Departamento de Odontopediatria, Ortodontia e Saúde Coletiva da FOB - USP. O período de aplicação do questionário foi de maio/2001 a outubro/2001.

\section{RESULTADOS}

O índice de retorno dos questionários foi de $39,5 \%$, ou seja, dos 248 questionários enviados, 98 foram devolvidos.
Dos 98 profissionais que responderam o questionário, 55 $(56,1 \%)$ eram do gênero masculino, enquanto que $43(43,9 \%)$ eram do feminino. Com relação ao estado onde clinicam, 74,7\% responderam ser em São Paulo, caracterizando a grande fixação destes cirurgiões-dentistas neste estado. Um ponto importante a se destacar é que os cirurgiões-dentistas que estão clinicando fora da região Sudeste são os que possuem as maiores rendas mensais nos consultórios particulares.

Os motivos que levaram o profissional a escolher a Odontologia como profissão podem ser observados na Figura 1.

As classificações atribuídas ao curso de Graduação foram, em ordem decrescente: Bom (54,1\%), Ótimo (43,9\%) e Regular (2,0\%). Apesar disso, 41,8\% afirmaram que 4 anos não são suficientes para o curso de Graduação em Odontologia, sendo que, destes, $90,3 \%$ indicaram que seriam necessários 5 anos, para que se pudesse aprofundar conhecimentos técnico-científicos $(65,9 \%)$, incorporar novas disciplinas na grade curricular $(24,4 \%)$ e para que houvesse aumento da carga horária $(31,7 \%)$.

Os resultados sobre as 5 disciplinas melhor abordadas e as 5 piores podem ser observados nas tabelas 1 e 2 .

Dos 98 cirurgiões-dentistas graduados de 1996 a 2000, participantes da pesquisa, 58,2\% fizeram ou fazem estágio, sendo que destes, $87,7 \%$ não eram remunerados. Pôde-se observar que $66,3 \%$ fizeram ou fazem Pós-Graduação em nível de Aperfeiçoamento/Especialização (52,3\%), Mestrado $(47,7 \%)$ e, em número menor, Doutorado $(7,7 \%)$. Esses profissionais, em sua maioria $(69,4 \%)$ consideram indispensável fazer cursos de Pós-Graduação, pois é uma tendência de mercado $(44,1 \%)$ que leva ao aprimoramento técnico-científico $(33,8 \%)$, melhora a qualificação profissional $(17,6 \%)$ e promove uma melhoria no atendimento clínico $(14,7 \%)$.

A Odontologia passa por uma fase em que os profissionais, principalmente os recém-formados, têm grande dificuldade para se estabelecer no mercado de trabalho, o que desestimula o exercício da profissão odontológica. Mesmo assim, 54,1\% responderam que se hoje tivessem que optar por uma carreira, escolheriam novamente a Odontologia, ao passo que $37,8 \%$ escolheriam outras carreiras, dentre as quais se destacaram a Medicina, a Administração e a área de Exatas. Embora 63,3\% se sintam realizados profissionalmente, apenas $12,2 \%$ se sentem realizados financeiramente.

O perfil predominante do profissional é aquele que trabalha no consultório particular próprio $(38,8 \%)$ ou trabalha por porcentagem $(25,5 \%)$, sendo que $26,5 \%$ atendem em algum Convênio ou Cooperativa e 12,2\% trabalham na rede pública. As 5 áreas em que estes cirurgiões-dentistas mais atuam no consultório são: Dentística $(59,2 \%)$, Cirurgia $(50,0 \%)$, Prótese $(39,8 \%)$, Periodontia $(32,7 \%)$ e Odontopediatria $(31,6 \%)$. Na clínica onde trabalham há outros dentistas atendendo $(59,2 \%)$ e/ou profissionais de outras áreas $(19,4 \%)$ como a Medicina (42,1\%), Fonoaudiologia $(42,1 \%)$ e Fisioterapia (31,6\%). A maioria não é assalariada $(69,4 \%)$ e dos que são $(24,5 \%), 50,0 \%$ recebem de $\mathrm{R} \$ 500,00$ a $\mathrm{R} \$ 990,00$ por mês. A renda mensal bruta predominante no 


\section{QUESTIONÁRIO}

Parte I: Dados pessoais

1. Data de nascimento:

2. Gênero:

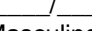

$1+1+$ Feminino

Idade: anos

3. Mês e ano em que se formou:

4. Cidade e Estado onde está clinicando:

Parte II: Perfil profissional

1. Por que você escolheu a Odontologia como profissão?

2. Qual classificação você atribui ao seu curso de Graduação?
( ) Péssimo
( ) Ruim
( ) Ótimo
( ) Bom
( ) Regular

3. Você acha que 4 anos são suficientes para o curso de Graduação em Odontologia?

( ) sim

( ) não. Na minha opinião anos são suficientes,

porque

4. Quais as matérias foram melhor abordadas e as que você considerou deficientes?

a)Melhores:

b) Piores:

Obs:

5. Faz ou já fez Estágio?

( ) não

( ) sim. Onde, em que área,

era remunerado?

6. Faz ou já fez algum curso de Pós-Graduação (Especialização, Mestrado, Doutorado,...)?

( ) não

( ) sim. Qual,

em que área e onde?

7. Acha indispensável fazer Pós-Graduação?
( ) não
( ) $\operatorname{sim}$

Porque

8. Hoje, se tivesse que optar por uma carreira, escolheria novamente a Odontologia?

( ) sim

( ) não. Escolheria fazer

porque

9. Você se sente profissionalmente realizado(a) como Cirurgião-Dentista?
( ) $\operatorname{sim}$
( ) não

Porque

10. Trabalha em (pode ser assinalada mais de uma opção)

( ) consultório particular próprio

( ) consultório particular de outro dentista, pagando um aluguel fixo

( ) consultório particular de outro dentista, pagando uma porcentagem sobre os procedimentos que realiza

( ) rede pública (prefeituras, p.ex.)

( ) não trabalho

( ) outro(s):

11. Atende em algum Convênio ou Cooperativa?
( ) não
( ) sim. Qual?

12. Realiza atividades de caráter educativo-preventivo no consultório?
( ) $\operatorname{sim}$
( ) não

13. Realiza atividades de caráter educativo-preventivo de maneira coletiva?

( ) $\operatorname{sim}($ ) não

14. Assinale com "+" as áreas em que você mais atua no seu consultório e com "“" as que você menos realiza ou não realiza:
( ) Dentística
( ) Periodontia
( ) Odontopediatria
( ) Endodontia
( ) Prótese
( Ortodontia

( ) Cirurgia

15. Na Clínica onde você trabalha (pode ser assinalada mais de uma opção):

( ) há outros dentistas atendendo

( ) há profissionais de outras áreas de saúde atendendo.

Qual(is) a(s) área(s)?

16. Você é assalariado?
( ) não
( ) sim. Recebo $R \$$ por mês.

17. Qual a sua renda no seu consultório?

a) Renda mensal bruta: $R \$$

b) Renda mensal líquida: R

18. Você se sente financeiramente realizado(a) como Cirurgião-Dentista?

( ) sim ( ) não

Porque

19. Quais as maiores dificuldades que você tem encontrado no exercício da Odontologia?

20. Quais as características que você julga serem necessárias aos Cirurgiões-Dentistas atualmente?

Os questionários foram tabulados e descritos por meio de tabelas e gráficos utilizando-se freqüência absoluta e relativa 


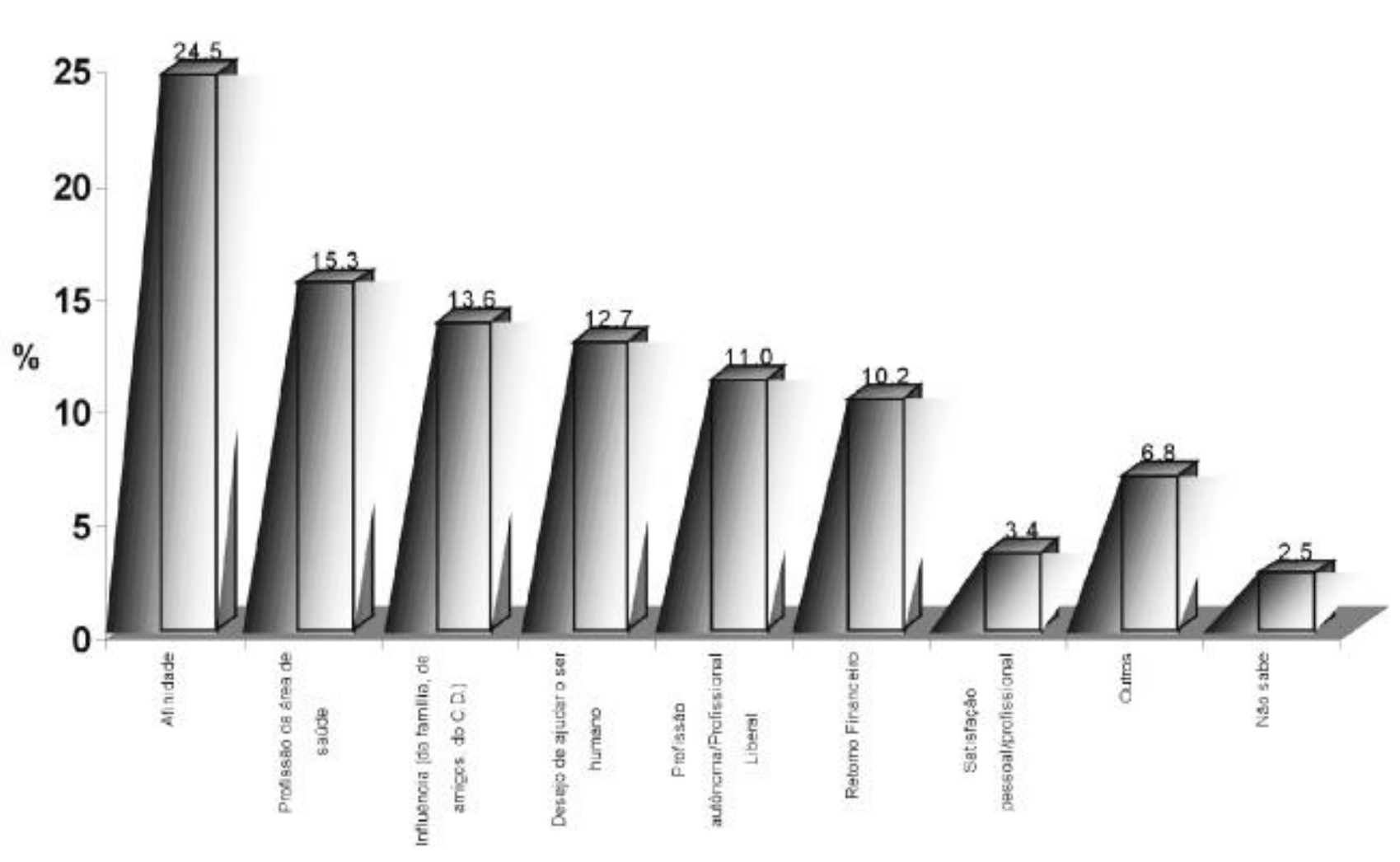

FIGURA 1- Motivos que levaram os profissionais a escolher a Odontologia como profissão

TABELA 1- Disciplinas melhor abordadas durante o curso de Graduação

\begin{tabular}{lll}
\hline DISCIPLINA & $\mathbf{n}$ & $\%$ \\
\hline Dentística & 78 & 79,6 \\
Matérias básicas & 54 & 55,1 \\
Endodontia & 35 & 35,7 \\
Cirurgia & 30 & 30,6 \\
Estomatologia & 26 & 26,5 \\
\hline
\end{tabular}

TABELA 3- As cinco maiores dificuldades que os cirurgiõesdentistas vêm encontrando no exercício da Odontologia

\begin{tabular}{lcc}
\hline DIFICULDADES & $\mathbf{n}$ & $\%$ \\
\hline $\begin{array}{l}\text { Mercado de trabalho } \\
\text { competitivo }\end{array}$ & 35 & 35,7 \\
$\begin{array}{l}\text { Pacientes não conseguem } \\
\text { pagar o tratamento }\end{array}$ & 17 & 17,3 \\
$\begin{array}{l}\text { Falta de ética e união entre a } \\
\text { classe odontológica }\end{array}$ & 14 & 14,3 \\
$\begin{array}{l}\text { Mercantilização e banalização } \\
\text { da Odontologia }\end{array}$ & 14 & 14,3 \\
Convênios/Clínicas populares & 13 & 13,3 \\
\hline
\end{tabular}


TABELA 4- As dez principais características que os participantes julgaram ser necessárias aos cirurgiões-dentistas atualmente

\begin{tabular}{llc}
\hline CARACTERÍSTICAS & $\mathbf{n}$ & \% \\
\hline Atualizar-se e estudar constantemente & 32 & 32,7 \\
Saber administrar, gerenciar e ter noções de Marketing & 23 & 23,5 \\
Ter conhecimento técnico-científico & 22 & 22,4 \\
Habilidade social/Boa relação interpessoal & 19 & 19,4 \\
Multidisciplinariedade e integração com as demais especialidades & 17 & 17,3 \\
e áreas da saúde & 10 & 10,2 \\
Amar a profissão & 10 & 10,2 \\
Ter paciência & 10 & 10,2 \\
Ser bom comerciante & 9 & 9,2 \\
Caráter e honestidade & 8 & 8,2 \\
Ter boa formação universitária & 8 \\
\hline
\end{tabular}

\section{DISCUSSÃO}

Do total de cirurgiões-dentistas graduados na FOB-USP entre 1996 e 2000, o índice de retorno de questionários, que ficou em $39,5 \%$, foi superior ao conseguido por $\mathrm{Costa}^{4}$ (7\%), mas inferior ao obtido por outros autores ${ }^{2,7,9}$. A alta taxa de perda, apesar de ser inerente ao tipo de metodologia utilizada, acabou por constituir uma limitação do presente estudo.

Quanto ao gênero dos profissionais, houve equilíbrio, com uma discreta predominância do gênero masculino $(56,1 \%)$. O que se observa é uma grande quantidade de cirurgiões-dentistas do gênero masculino nos trabalhos mais antigos: Costa $^{4}$ (69\%); Falasco, Robinson, Faja $^{7}$ (90\%); Greenwood, Lewis, Burgess ${ }^{9}$ (64,3\%); Stewart, Ralph, Macmillan ${ }^{16}$ (85\%). Contudo, Costa ${ }^{4}$, em 1992, já afirmava que o número de cirurgiãs-dentistas vinha aumentando rapidamente desde a década de 70 , o que com certeza, implicaria em mudanças profundas na profissão, pois as profissionais apresentam clara tendência ao trabalho assalariado e têm sua atuação clínica calcada mais sobre a Odontopediatria e Endodontia. Este aumento de profissionais de gênero feminino foi constatado nos trabalhos de Carvalho, Carvalho' 1997 e Marcelino $^{13}, 2000$.

Os motivos que levaram os participantes a escolher a Odontologia como profissão giram em torno de afinidade/ vocação, o fato de ser uma profissão da área de saúde, ou mesmo por influência da família, de amigos ou de cirurgiõesdentistas ou o desejo de ajudar o ser humano. Em menor percentual apareceram o fato de ser uma profissão liberal, o retorno financeiro e satisfação pessoal/profissional. Estes resultados vão de encontro aos achados de Carvalho e Carvalho $^{1}$, Costa $^{4}$ e Marcelino ${ }^{13}$.

A sugestão para aumento da carga horária parece ser um consenso entre cirurgiões-dentistas que participaram de diversos estudos $5,9,10,11,15,17$. As disciplinas que são melhor abordadas durante o curso de Graduação são aquelas referentes às atividades mais freqüentes na prática Odontológica, como Dentística, Endodontia e Cirurgia, corroborando o estudo de Costa ${ }^{5}$. Já as disciplinas consideradas abordadas de maneira deficiente também foram semelhantes aos achados de outros autores ${ }^{5,17}$. Os resultados da pesquisa de Greenwood, Lewis, Burgess ${ }^{9}$ demonstraram que as competências em que os profissionais se sentiam menos preparados foram: manejo do paciente, e os aspectos legais, éticos e administrativos. A administração das finanças e o tratamento de dor orofacial crônica, que consistiram nas maiores deficiências do profissional, são resultados similares a outros estudos ${ }^{8,10,11,15,17}$. Vários trabalhos ${ }^{8,11,15}$ semelhantes a este apontam que os profissionais saem da Faculdade bem preparados nas atividades mais comuns da profissão, como dentística, exame clínico, diagnóstico, plano de tratamento, anestesia local e raspagem periodontal.

O percentual de profissionais que fizeram ou fazem PósGraduação atingiu os 66,3\%. Para $\mathrm{Costa}^{3}$, a procura por cursos diversos após a graduação está associada à precariedade da formação, muito mais que um esforço de atualização. $\mathrm{O}$ autor também citou Madeira e Carvalho ${ }^{12}$, os quais afirmaram ser responsabilidade da faculdade estabelecer as características do profissional por ela formado, definindo-o segundo critérios claros, distinguindo entre o que é preciso ser ensinado do que deve ser ensinado.

Em concordância com Costa ${ }^{4}$, Marcelino ${ }^{13}$ e Stewart, Ralph, Macmillan ${ }^{16}$, a análise do perfil profissional mostrou a predominância de cirurgiões-dentistas trabalhando no consultório particular, sendo que Dentística, Cirurgia, Prótese, Periodontia e Odontopediatria são as áreas de maior atuação.

$\mathrm{O}$ alto percentual de profissionais trabalhando com outros cirurgiões-dentistas representa um fator positivo ressaltado por Falasco, Robinson, $\mathrm{Faja}^{7}$, pois os autores observaram que aqueles que trabalham em grupo apresentam menores dificuldades em relação aos que trabalham sozinhos. Assim como relatou Marcelino ${ }^{13}$, há uma integração com outros profissionais de saúde, principalmente médicos, fonoaudiólogos e fisioterapeutas.

Embora a maioria tenha afirmado estar realizada profissionalmente, houve um alto percentual de insatisfação financeira (80,6\%), até maior do que encontrado por Costa ${ }^{4}$, o qual também observou que o ápice na curva de 
remuneração coincidiu com o maior sentido de realização. O autor se surpreendeu pelo fato de que, mesmo neste ponto, os percentuais de realização plena foram relativamente baixos, bem como pela forte corrente de insatisfação financeira que perdura e cresce sob todas as faixas etárias.

Os resultados demonstram que os cirurgiões-dentistas ainda não despertaram para a importância da educação e da prevenção, principalmente em nível coletivo.

Dentre as maiores dificuldades enfrentadas pelos cirurgiões-dentistas, a alta competitividade e saturação do mercado de trabalho, bem como o baixo poder aquisitivo da população também foram observados por outros autores ${ }^{7,13}$

Uma das principais características que os participantes julgaram ser necessárias aos cirurgiões-dentistas são noções de Administração, Gerenciamento e Marketing. Já em 1979, Dean $^{6}$, através de uma pesquisa na Faculdade de Odontologia da Universidade do Alabama, EUA, constatou que os estudantes não se sentiam capacitados a gerenciar um consultório, devido, principalmente, à falta de base pela abordagem deficiente desta Disciplina durante o curso de graduação. Falasco, Robinson, Faja ${ }^{7}, 1990$, afirmaram que a Administração e o Gerenciamento do consultório são fundamentais, devido ao aumento da competitividade, aumento no número de profissionais, surgimento de novas tecnologias e os altos custos das clínicas. Assim, a partir dos resultados dos questionários aplicados a recémformados da Faculdade de Odontologia de Michigan, EUA, concluíram que em tempos de recessão e alta competitividade, para se alcançar o sucesso na clínica odontológica, faz-se necessária a utilização efetiva de estratégias de Marketing, para que o estabelecimento e a manutenção do consultório se tornem viáveis. Costa ${ }^{5}, 1992$, constatou que o ensino da administração do consultório deveria ser incrementado, deixando claro que a faculdade tem sido ineficiente, senão negligente, em atender ao seu caráter profissionalizante no seu ponto mais crítico.

\section{CONCLUSÕES}

Considerando os cirurgiões-dentista graduados na FOBUSP entre 1996 e 2000 que participaram do presente estudo, é possível concluir que:

1. Os principais motivos que os levaram a escolher a Odontologia como profissão foram: afinidade, o fato de ser uma profissão da área de saúde, influência da família, de amigos ou do cirurgião-dentista e o desejo de ajudar o ser humano.

2. O curso de Graduação foi classificado como tendo sido bom pela maior parte dos respondentes, apesar da maioria haver sugerido o aumento da carga horária.

3. As 5 Disciplinas melhor abordadas durante a Graduação foram: Dentística, Matérias básicas, Endodontia, Cirurgia e Estomatologia. As 5 consideradas ministradas de maneira deficiente foram: Ortodontia, Odontologia Preventiva/Saúde Coletiva, Prótese, Matérias básicas e Periodontia.
4. Grande parte fez ou faz cursos de Pós-Graduação.

5. O perfil predominante do profissional é aquele que trabalha no consultório particular próprio ou trabalha por porcentagem, sendo que $26,5 \%$ atendem em algum Convênio ou Cooperativa e $12,2 \%$ trabalham na rede pública.

6. As 5 áreas em que estes cirurgiões-dentistas mais atuam no consultório são: Dentística, Cirurgia, Prótese, Periodontia e Odontopediatria.

7. A maioria não é assalariada e dos que são, metade recebe de $\mathrm{R} \$ 500,00$ a $\mathrm{R} \$ 990,00$ por mês. A renda mensal bruta predominante no consultório é de $\mathrm{R} \$ 1.100,00$ a $\mathrm{R} \$ 2.000,00$, enquanto que a renda mensal líquida da maioria dos profissionais é de até $\mathrm{R} \$ 1.000,00$.

8. Apesar de uma parcela considerável ter afirmado se sentir realizado profissionalmente, a taxa de insatisfação financeira foi alta.

9. Os cirurgiões-dentistas ainda não despertaram para a importância da educação e da prevenção, principalmente em nível coletivo.

10. Uma das maiores dificuldades enfrentadas pelos cirurgiões-dentistas é a alta competitividade do mercado de trabalho.

\section{ABSTRACT}

The aims of this study were to determine the professional profile of the 248 dentists graduated at Bauru Dental School - University of Sao Paulo between 1996 and 2000, verify if they accomplish educational and collective measures and determine the level of professional satisfaction. The material was a self-applicable questionnaire containing both multiple choice and open questions. The return rate was $39.5 \%$. Analysis of data showed that the dentists, who were predominantly males, chose Dentistry as a profession because they felt comfortable with it, and evaluate the graduate course as good. Results showed that the practitioners still do not focus on the need of education and prevention in oral health, especially at the collective level. It was concluded that the predominant professional profile was to work at their dental office $(38.8 \%)$ or at a peer's dental office, receiving an amount of the total profile (25.5\%); $26.5 \%$ work with health mutual support and $12.2 \%$ work at public service. Although $63.3 \%$ are professionally realized, only $12.2 \%$ are economically realized. The great difficult is the increase in the competition between dentists and saturation of the dental work market.

UNITERMS: Dental staff; Professional practice; Dentists; Questionnaires.

\section{REFERÊNCIAS BIBIOGRÁFICAS}

1- Carvalho DR de, Carvalho ACP de. Motivações e expectativas para o curso e para o exercício da Odontologia. Estudo com formandos da capital de São Paulo. São Paulo : NUPES; 1997. 
2- Chambers DW, Eng Junior WR. Practice profile: the first twelve years. J Calif Dent Assoc 1994; 22:25-32.

3- Costa B. Do ensino à prática odontológica: mito e realidade na grande São Paulo. São Paulo ; 1988. [Tese de Doutorado Faculdade de Odontologia da USP].

4- Costa B, Stegun RC, Todescan R. Realização profissional: uma avaliação entre os dentistas na Grande São Paulo. Rev Assoc Paul Cir Dent 1992, 46:821-4.

5- Costa B, Stegun RC, Todescan R. Do ensino à prática odontológica: um levantamento da realidade na Grande São Paulo. Rev Assoc Paul Cir Dent 1992, 46:909-13.

6- Dean DH. Are dental students prepared to manage their practices? Dent Stud 1979, 58:21-2.

7- Falasco RF, Robinson E, Faja BW. Problems encountered by recent graduates in establishing dental practices. J Mich Dent Assoc 1990; 72:15-9.

8- Gerbert B, Badner V, Maguire B, Martinoff J, Wycoff S, Crawford W. Recent graduates' evaluation of their dental school education. J Dent Educ 1987; 51:697-700.

9- Greenwood LF, Lewis DW, Burgess RC. How competent do our graduates feel? J Dent Educ 1998, 62:307-13.

10- Haug SP, Brown DT, Goodacre CJ, Cerimele BJ. Recent graduates' and current dental students' evaluation of their prosthodontic curriculum. J Prosthet Dent 1993, 70:361-71.

11- Holmes DC, Diaz-Arnold AM, Williams VD. Alumni selfperception of competence at time of dental school graduation. J Dent Educ 1997, 61:465-72.

12- Madeira MC, Carvalho ACP. Necessidades e tendências da Odontologia nas faculdades e no exercício da profissão. A propósito de uma enquete. Rev Assoc Paul Cir Dent 1980, 34(4):284-93.

13- Marcelino G. Avaliação do perfil profissional e percepção social de cirurgiões-dentistas do município de Araçatuba-SP frente aos avanços ocorridos na odontologia às vésperas do século XXI. Araçatuba ; 2000. [Dissertação de Mestrado - Faculdade de Odontologia de Araçatuba da UNESP].

14- ODONTOLOGIA do Brasil faz 117 anos e avança no século XXI. Disponível na Internet. http://www.jornalexpress.com.br/ noticias/detalhes.php?id_jornal=848\&id_noticia=153. 04 set. 2003.

15- Solomon ES, Whiton MS. Dental seniors' evaluation of their curriculum and the number of clock hours of instruction. J Dent Educ 1991, 55:743-5.

16-Stewart BL, Ralph WJ, Macmillan CH. Survey of dental practice/dental education in Victoria. Part I. Questionnaire/general aspects. Aust Dent J 1989; 34:563-70.

17- Stewart BL, Ralph WJ, Macmillan CH. Survey of dental practice/dental education in Victoria. Part II. Recent graduates/ graduating students. Aust Dent J 1990; 35:69-75.
18-Stewart BL, Ralph WJ, Macmillan CH. Survey of dental practice/dental education in Victoria. Part III. Trends in general dental practice. Aust Dent J 1990; 35:169-80.

Recebido para publicação em: 07/06/2002

Enviado para reformulações em: 26/07/2002

Pronto para publicação em: 10/09/2003

Recebido para publicação em: 07/06/2002

Enviado para reformulações em: 26/07/2002

Pronto para publicação em: 10/09/2003

\section{ENDEREÇO DOS AUTORES PARA CORRESPONDÊNCIA}

Prof. Dr. José Roberto de Magalhães Bastos

Faculdade de Odontologia de Bauru - USP

Departamento de Odontopediatria, Ortodontia e Saúde Coletiva

Área de Saúde Coletiva

Al. Dr. Octávio Pinheiro Brisolla, 9-75

17012-901 BAURU, SP

Fone: (14) 235-8257

E-mail: jrbastos@fob.usp.br 